This information is current as of April 26, 2023.

\title{
Neuronavigation-Guided Focused Ultrasound-Induced Blood-Brain Barrier Opening: A Preliminary Study in Swine
}

K.-C. Wei, H.-C. Tsai, Y.-J. Lu, H.-W. Yang, M.-Y. Hua, M.-F. Wu, P.-Y. Chen, C.-Y. Huang, T.-C. Yen and H.-L. Liu

AJNR Am J Neuroradiol 2013, 34 (1) 115-120 doi: https://doi.org/10.3174/ajnr.A3150

http://www.ajnr.org/content/34/1/115 


\title{
Neuronavigation-Guided Focused Ultrasound-Induced Blood- Brain Barrier Opening: A Preliminary Study in Swine
}

\author{
K.-C. Wei, H.-C. Tsai, Y.-J. Lu, H.-W. Yang, M.-Y. Hua, M.-F. Wu, P.-Y. Chen, C.-Y. Huang, T.-C. Yen, and H.-L. Liu
}

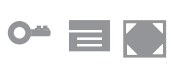

\begin{abstract}
BACKGROUND AND PURPOSE: FUS-induced BBB opening is a promising technique for noninvasive and local delivery of drugs into the brain. Here we propose the novel use of a neuronavigation system to guide the FUS-induced BBB opening procedure and investigate its feasibility in vivo in large animals.
\end{abstract}

MATERIALS AND METHODS: We developed an interface between the neuronavigator and FUS to allow guidance of the focal energy produced by the FUS transducer. The system was tested in 29 swine by more than 40 sonication procedures and evaluated by MR imaging. Gd-DTPA concentration was quantitated in vivo by MR imaging RI relaxometry and compared with ICP-OES assay. Brain histology after FUS exposure was investigated using H\&E and TUNEL staining.

RESULTS: Neuronavigation could successfully guide the focal beam, with precision comparable to neurosurgical stereotactic procedures $(2.3 \pm 0.9 \mathrm{~mm})$. A FUS pressure of $0.43 \mathrm{MPa}$ resulted in consistent BBB opening. Neuronavigation-guided BBB opening increased Gd-DTPA deposition by up to $1.83 \mathrm{mmol} / \mathrm{L}$ (a $140 \%$ increase). MR relaxometry demonstrated high correlation with ICP-OES measurements $\left(r^{2}=\right.$ 0.822), suggesting that Gd-DTPA deposition can be directly measured by imaging.

CONCLUSIONS: Neuronavigation provides sufficient precision for guiding FUS to temporally and locally open the BBB. Gd-DTPA deposition in the brain can be quantified by MR relaxometry, providing a potential tool for the in vivo quantification of therapeutic agents in CNS disease treatment.

ABBREVIATIONS: CE = contrast-enhanced; FUS = focused sonography; Gd-DTPA = gadolinium-diethylene-triamine pentaacetic acid; ICP-OES = inductively coupled plasma optical emission spectroscopy; MRgFUS = MR-guided FUS; SI = signal intensity; TUNEL = terminal deoxynucleotidyl transferase biotin-dUTP nick end-labeling

$\mathbf{T}$ he BBB prevents molecules larger than 400 Da from entering the brain parenchyma, protecting it from toxic foreign substances and preventing entry of therapeutic drugs. ${ }^{1,2}$ The perme-

Received February 17, 2012; accepted after revision March 14, 2012.

From the Departments of Neurosurgery (K.-C.W., H.-C.T., Y.-J.L., P.-Y.C., C.-Y.H.), Nuclear Medicine (T.-C.Y.), Chemical and Material Engineering (Y.-J.L., H.-W.Y., M.Y.H., P.-Y.C.), and Electrical Engineering (H.-L.L.), Molecular Imaging Center (T.-C.Y., H.-L.L.), Healthy Aging Research Center (H.-L.L.), and Graduate Institute of Clinical Medical Sciences (H.-C.T.), Chang-Gung University and Memorial Hospital, Taoyuan, Taiwan; and Animal Medicine Center (M.-F.W.), National Taiwan University, Taipei, Taiwan.

This project was supported by the National Science Council, Taiwan (NSC-992321-B-182-008, NSC-100-2120-M-182A-001), Chang-Gung Memorial Hospital, Taiwan (CMRPG3A0531, CMRPG3A0501), and National Health Research Institutes, Taiwan (NHRI-EX101-10004N).

Please address correspondence to Hao-Li Liu, PhD, Associate Professor, Department of Electrical Engineering, Chang-Gung University, 259 Wen-Hwa lst Rd, Kweishan,Taoyuan, 333 Taiwan; e-mail: haoliliu@mail.cgu.edu.tw

- Indicates open access to non-subscribers at www.ajnr.org

$\equiv$ Indicates article with supplemental on-line tables.

Indicates article with supplemental on-line figures.

http://dx.doi.org/10.3174/ajnr.A3150 ability of the BBB can be increased by FUS using microbubbles and a low-energy burst tone. ${ }^{3,4}$ This noninvasive procedure disrupts the BBB locally, rather than systemically, to minimize offtarget effects, and disruption is reversible within several hours. $\mathrm{BBB}$ opening can be confirmed by increased permeability of MR imaging contrast agents such as Gd-DTPA ${ }^{3-5}$ or superparamagnetic iron oxide nanoparticles. ${ }^{6}$

Sonography focal energy needs to be guided with high precision to open the BBB at a specific target. One successful approach is MRgFUS, originally designed for monitoring and guiding thermal ablation by an embedded FUS system inside the MR bore. Brain tissues have been successfully ablated transcranially by monitoring the temperature response in both large animals ${ }^{7}$ and humans ${ }^{8,9}$; however, widespread use of MRgFUS is hampered by its design complexity and cost. In addition, MR scanners block access of neurosurgeons to patients, and most surgical tools were not designed to be MR compatible. Although the focal beam can be localized by the slight temperature elevation caused by weak, continuous wave exposure, actual BBB opening can currently 
only be confirmed by MR contrast agent leakage after, but not during, the sonication process. ${ }^{3,10}$ Thus, despite the successful demonstration of FUS BBB opening in large animals, MR imaging has only served as a postoperative verification tool and has not addressed the issue of guidance of FUS energy during the procedure. ${ }^{1-13}$ An alternative approach is therefore needed to integrate FUS as one of an array of available neurosurgical tools while preserving the excellent capability of precise focal beam guidance of the MRgFUS system.

The neuronavigational guidance system has revolutionized neurosurgery by providing a way to navigate through the body using 3D images. ${ }^{14}$ Preoperative diagnostic scans of patients (usually CT images or MR imaging) are first analyzed, followed by a registration process that allows $3 \mathrm{D}$ localization of the surgical tools and that assists neurosurgeons in mapping the safest, least invasive path to the target site for tumor removal surgery, interstitial interventions, or biopsy. Currently, however, only standard solid surgical instruments, such as biopsy needles, catheters, or microsurgical robots, can be guided, as neuronavigation of the focal energy generated by a FUS transducer in CNS tissues has not been attempted.

The purpose of our study was to design a procedure for neuronavigation-guided FUS brain drug delivery by guiding the focal beam and to investigate its feasibility in vivo in large animals (swine, Sus scrofa domestica).

\section{MATERIALS AND METHODS}

\section{Neuronavigation Guidance Setup}

We used a neuronavigation system (Stryker, Freiburg, Germany) to guide FUS BBB opening. The procedure was initiated by registering the focal point of the FUS transducer on the neuronavigation system. A sonography-permeable balloon-type membrane was then tightly fitted to the sonography transducer to fully cover the active elements and couple the sonography energy from the transducer element to the animal brain (the detailed procedure is provided in On-line Fig 1).

\section{Focused Sonography Exposure}

Animals were anesthetized by isofluorane. The top of the cranium was shaved and a PE-50 catheter (Instech Labs, Plymouth Meeting, Pennsylvania) was inserted into the ear vein for injections. During FUS treatment, sonography microbubbles were constantly infused through the ear vein (SonoVue; Bracco Diagnostic, Milan, Italy) at $0.3 \mathrm{~mL} / \mathrm{kg} / \mathrm{min}$. Sonography was delivered transcranially by a spherical single-element transducer (Imasonics, Besancon, France; diameter $=60 \mathrm{~mm}$, radius of curvature $=$ $80 \mathrm{~mm}$, frequency $=400 \mathrm{kHz}$; measured half-maximum pressure amplitude diameter approximately $4 \mathrm{~mm}$; length of the produced focal spot approximately $23 \mathrm{~mm}$ ). Animal experiments were divided into 4 groups (On-line Table 1). In groups 1,2 , and 3 ( $n=$ 5,9 , and 9 , respectively), we evaluated the accuracy of neuronavigation-guided $\mathrm{BBB}$ opening with different negative peak pressures $(0.26,0.43$, and $0.56 \mathrm{MPa}$, respectively, after considering skull and tissue decay) using a single FUS exposure. This experiment also allowed us to determine the BBB-opening threshold pressure. FUS energy was delivered in burst-tone mode, with burst length $=10 \mathrm{~ms}$, pulse-repetition frequency $=1 \mathrm{~Hz}$, and sonication duration $=30$ seconds. In group $4(n=6)$, we investigated whether we could also obtain large BBB-opened regions with expected target volumes by precise neuronavigational guidance of multipoint FUS exposures $(3 \times 3$ arrangement, $5-\mathrm{mm}$ spacing; 0.43-MPa sonication). All animals were humanely killed within 24 hours.

Calibrated ultrasonic pressure and focal beam dimensions were measured both with and without a harvested swine skull, and the pressure loss caused by skull distortion was estimated to be $30 \%$, with no apparent focal beam shift (On-line Fig $1 C$, -D). Transcranial pressure loss measured in the human skull was approximately $40 \%$ (data not shown).

\section{MR Imaging}

MR imaging was conducted with a 3T MR imaging scanner (Trio with Tim; Siemens, Erlangen, Germany). All animals underwent an initial MR imaging scan for setup, registration, and treatment planning for the neuronavigation system, and another scan to evaluate the treatment outcome. Animals were placed in a homemade stereotactic frame mounted on a position-fixed deck to guarantee identical imaging orientations for MR imaging scans.

Animals were anesthetized by intraperitoneal injection of chlorohydrate $(30 \mathrm{mg} / \mathrm{kg})$. Gd-DTPA (0.1 mmol/kg; Magnevist; Berlex Laboratories, Wayne, New Jersey) was administered, followed by saline $(0.2 \mathrm{~mL} / \mathrm{kg})$ and heparin $(0.2 \mathrm{~mL} / \mathrm{kg})$. T1-weighted MR images were acquired immediately after the completion of Gd-DTPA administration (TR/TE $=700 \mathrm{~ms} / 11 \mathrm{~ms})$, followed by acquisition of T2-weighted images (TR/TE $=3260 \mathrm{~ms} / 96 \mathrm{~ms})$.

A heavy $\mathrm{T}^{*}$-weighted fast low-angle shot sequence with full flow compensation in all 3 directions (TR/TE/flip angle $=28 \mathrm{~ms} / 20 \mathrm{~ms} /$ $15^{\circ}$ ) was modified to produce SWI for identifying intracerebral hemorrhaging caused by excessive FUS exposure. ${ }^{15}$

R1 relaxivity was quantified by transferring signal intensities from 2 T1-weighted gradient recalled-echo images with different flip angles, as previously described $(\mathrm{TR} / \mathrm{TE}=101 \mathrm{~ms} / 4.1 \mathrm{~ms}$, flip angle $=$ $\left.20^{\circ} / 40^{\circ}\right) .{ }^{16,17}$ In vitro phantom measurements showed that the R1 relaxation rate was linearly proportional to Gd-DTPA concentration (On-line Fig 2). These measurements were used to calibrate the R1 relaxivity rate (in seconds ${ }^{-1}$ ) and estimate $\mathrm{R} 1$ relaxivity (3.821 $\mathrm{mmol} / \mathrm{L}^{-1}$ second $^{-1}$ ). Identical MR imaging sequence setups were then applied to the animal MR imaging scans. We also quantitated Gd-DTPA deposition in the BBB-opened brain by ICP-OES assays and compared our findings with R1 relaxometry (see On-line materials).

\section{Evaluation of Accuracy of Neuronavigation Guidance}

The accuracy of neuronavigation in guiding FUS BBB opening was quantified by measuring the discrepancy between the assigned and actual BBB opening location on the focal plane.

\section{Histologic Examination}

Evans blue dye was administered after MR imaging for gross observation of the BBB-disrupted region. Animals were sacrificed and brain tissue samples from the sonicated sites and contralateral (control) hemispheres were fixed in formaldehyde. In each animal, 5-20 frozen sections (10- $\mu \mathrm{m}$ thick) covering the FUS exposure regions were stained by hematoxylin and eosin to assess hemorrhagic dam- 


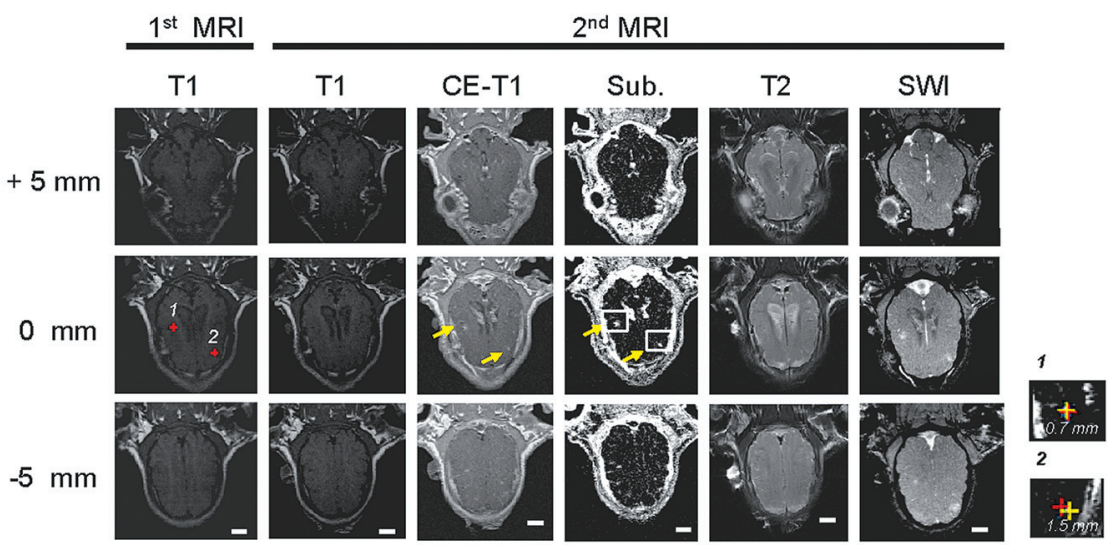

FIG 1. Typical MR image sets (0.43-MPa pressure). Column 1: T1-weighted images obtained in the first MR imaging scan (before FUS exposure, for neuronavigation guidance). Columns 2-4: T1, CE-T1, and subtracted T1-weighted images obtained in the second MR imaging scan (after FUS exposure, for treatment validation). Column 5: T2-weighted images. Column 6: Susceptibilityweighted images. Zoomed regions (right) demonstrate the measured discrepancy between the targeted and maximum SI increase locations at targets 1 and 2 ( 0.7 and $1.5 \mathrm{~mm}$, respectively). the BBB-opened location for all experiments (0.43- $\mathrm{MPa}$ and $0.56-\mathrm{MPa}$ exposures) was $2.3 \pm 0.9 \mathrm{~mm}$ (Fig $3 A$ ), which is within the acceptable range of clinical neuronavigational guidance.

We investigated the effect of different pressure levels on BBB opening (Fig 3B). Gd-DTPA contrast enhancement suggested consistent $\mathrm{BBB}$ opening at a pressure of $0.43 \mathrm{MPa}$ (SI increase $=17.9 \pm$ $11.6 \%$ ) but not at $0.26 \mathrm{MPa}$ (SI increase $=$ $-0.3 \pm 10.9 \%)$. Further increasing the FUS pressure to $0.56 \mathrm{MPa}$ provided higher contrast enhancement (SI = $28.1 \pm 23.8 \%)$. We also measured the change of relaxation rate caused by $\mathrm{BBB}$ opening. An exposure of $0.26 \mathrm{MPa}$ failed to produce any apparent change in the $\mathrm{R} 1$ relaxivity rate $\left(-0.15 \pm 0.45\right.$ seconds $\left.^{-1}\right)$, whereas higher pressure levels of 0.43 or age. Another 3-6 sections were stained by TUNEL assay (ApopTag kit; Intergen, New York, New York). Histologically confirmed tissue damage was characterized by various grades of increasing damage. $^{15,18}$

\section{RESULTS}

A typical MR imaging result is shown in Fig 1. The identical image orientation of the first and second T1 scans (Fig 1, columns 1 and 2) suggested minimal animal movement in the stereotactic holder. The positions of 2 selected FUS exposure locations (yellow markers) were entered into the neuronavigation system for focalbeam guidance. The BBB-opened regions were clearly visible in the T1, CE-T1, and subtracted images (Fig 1, columns 2-4) of the second scan but quickly disappeared when leaving the focal depth, suggesting that they were close to the planned depth of treatment. The discrepancies between the BBB-opened regions and the planned target points 1 and 2 were 0.7 and $1.5 \mathrm{~mm}$, respectively, in the coronal focal plane (Fig 1, zoomed images), indicating that neuronavigation successfully guided the focal beam to the targets. The unchanged T2-weighted images (Fig 1, column 5) showed that there was no brain edema for this treatment exposure. Moreover, the lack of signal decay in the $\mathrm{T} 2{ }^{*}$ image (Fig 1, column 6 ) indicated that the intracerebral hemorrhage had not occurred during sonication. The slight signal increase at the BBB-opened regions was probably caused by Gd-DTPA leakage.

We compared the R1 map results of the brain before and after FUS exposure and Gd-DTPA injection (Fig 2A). The BBB-opened regions showed a high $\mathrm{R} 1$ relaxation rate $\left(>4\right.$ seconds $^{-1}$ compared with $<2$ seconds $^{-1}$ in the contralateral brain). From the Gd-DTPA relaxivity, we estimated that Gd-DTPA was deposited at a concentration of more than $1.05 \mathrm{mmol} / \mathrm{L}$ in the BBB-opened region, compared with less than $0.52 \mathrm{mmol} / \mathrm{L}$ in the contralateral brain. FUS exposure thus enabled leakage of at least $0.5 \mathrm{mmol} / \mathrm{L}$ Gd-DTPA into brain parenchyma. These contrast enhancement areas were supported by the distribution of Evans blue dye in comparable brain sections (Fig $2 B$ ).

The mean distance discrepancy between the target point and
$0.56 \mathrm{MPa}$ produced significant monotonic increases in relaxivity rates-1.15 \pm 0.95 seconds $^{-1}(26.6 \pm 19.9 \%$ to contralateral brain) and $2.45 \pm 1.65$ seconds $^{-1}(79.0 \pm 36.9 \%$ to contralateral brain), respectively (Fig 3C) - equivalent to 0.3 and $0.6 \mathrm{mmol} / \mathrm{L}$ of Gd-DTPA permeating into brain parenchyma. Thus, neuronavigation could provide sufficient precision in guiding FUS to temporally and locally open the blood-brain barrier, and GdDTPA deposition of up to $1.83 \mathrm{mmol} / \mathrm{L}$ ( $140 \%$ increase) could be achieved by $0.56 \mathrm{MPa}$ sonication.

We measured the correlation between Gd-DTPA contrast-enhanced signal changes in MR imaging, and Gd-DTPA concentrations measured by ICP-OES assay, for 36 tissue samples obtained from 2 animals (Fig $3 E$ ). The T1 contrast-enhanced change was well correlated with the measured gadolinium concentration $\left(r^{2}\right.$ $=0.598$ ) and could therefore provide semiquantitative information on gadolinium deposition in the BBB-opened brain. However, R1 maps were found to provide direct in vivo quantification of the amount of Gd-DTPA leaked into the BBB-opened region and also showed improved correlation with the measured concentrations (Fig $3 F ; r^{2}=0.822$ ).

Next we showed that a large BBB-opened region could be successfully created by multipoint FUS exposure performed in a $3 \times 3$ shape, with 5-mm spacing between each sonication point (Fig $2 C$; group 4 animals). The diameter of the opening was significantly enlarged (approximately $20 \mathrm{~mm}$ ) in the coronal plane compared with single-point sonication $(4 \mathrm{~mm})$. A brain section of the same animal confirmed significant enlargement of BBB opening (Fig 2D).

Tissues from regions exposed to FUS at different levels of pressure were histologically classified (On-line Fig 3). The 0.26-MPa exposure did not consistently introduce a BBB-opening effect and a most of the samples were unchanged upon H\&E staining. Some samples had vasodilations. Tissues from the 0.43-MPa exposure were also mostly intact, with occasional nongrouping erythrocyte extravasations (8.6\%). Grouping extravasations increased by $3.1 \%$ for $0.56-\mathrm{MPa}$ exposure, and further increased to $6.4 \%$ in these perivascular hemorrhage locations showed signal changes in multiple $0.43-\mathrm{MPa}$ sonication exposures. Nevertheless, none of 
$+5 \mathrm{~mm}$

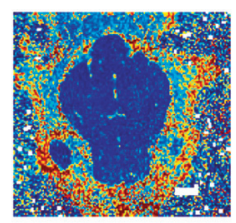

A

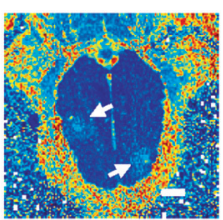

$-5 \mathrm{~mm}$

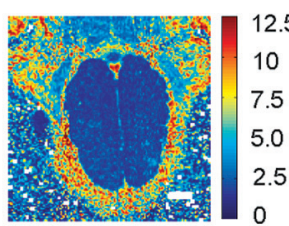

$+5 \mathrm{~mm}$

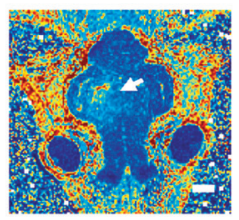

B
$0 \mathrm{~mm}$

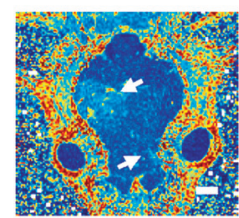

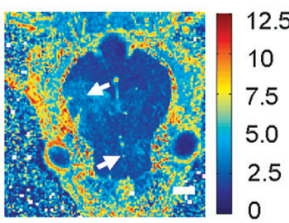
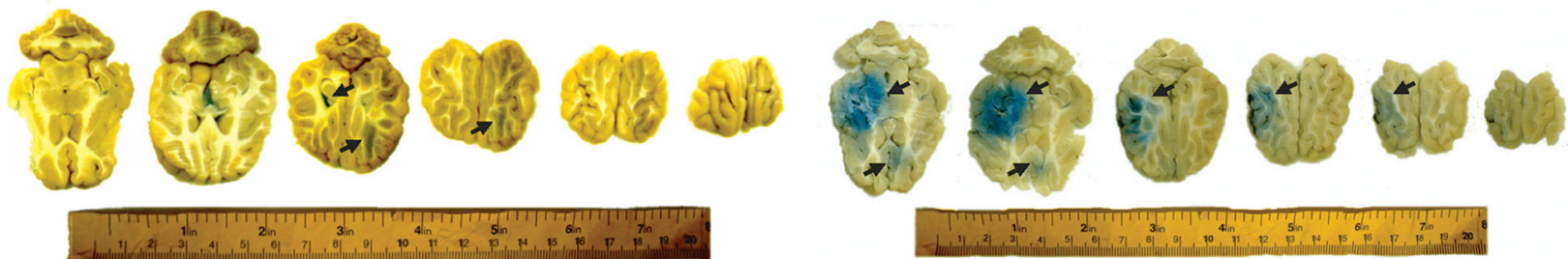

C

FIG 2. R1 maps and the Evans blue-stained brain sections obtained from single-point $(A, B$; same animal as Fig 1$)$ and multipoint ( $3 \times 3)$ FUS exposures $(C, D)$. Arrows denote significant R1 $(A, B)$ or Gd-DTPA leakage regions $(C, D)$. Size bar $=10 \mathrm{~mm}$.

A

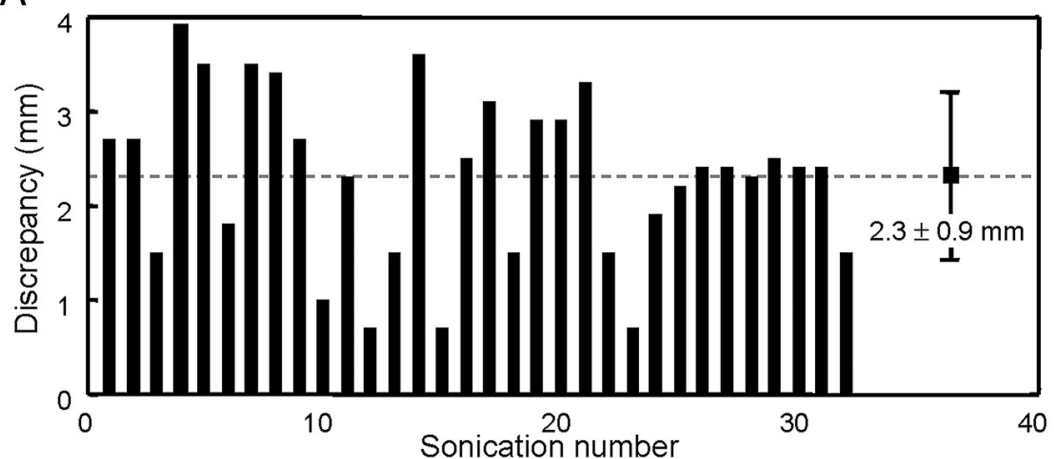

B

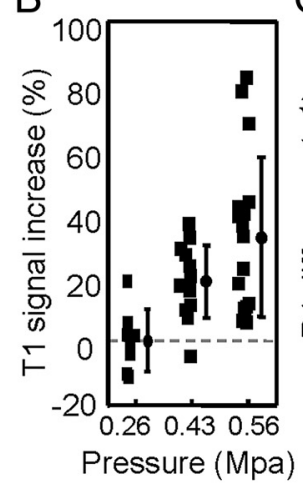

C

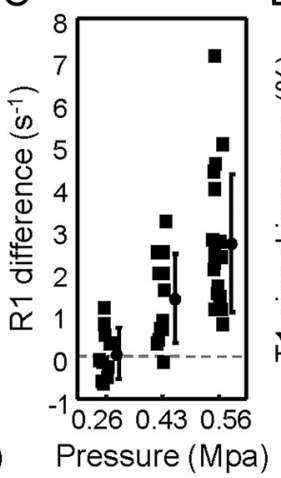

D

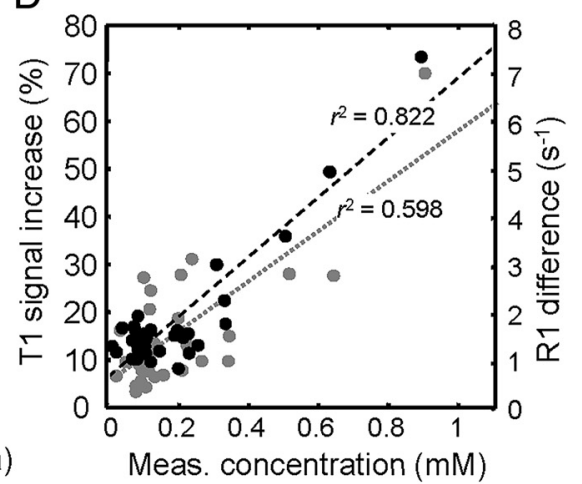

FIG 3. A, Discrepancy (in $\mathrm{mm}$ ) between the planned target point and BBB-opening points measured from subtracted CE-TT-weighted images of all experiments. The average discrepancy was $2.3 \pm 0.9 \mathrm{~mm}$. B, MR imaging signal intensity increase obtained from the signal differences between T1- and CE-TT-weighted images at the sonication target after different FUS exposure levels $(0.26,0.43$, and $0.56 \mathrm{MPa}) . C$, The difference (in seconds ${ }^{-1}$ ) between R1 measurements of the first and the second (postoperative) MR imaging scans under different FUS pressures $(0.26$, 0.43 , and $0.56 \mathrm{MPa}$ ). $D$, Correlation between the measured Gd concentration (in $\mathrm{mM}$; from ICP-OES assay; gray marker) and the SI contrast enhancement (in \%) obtained from T1 images, and correlation between the measured Gd concentration (in $\mathrm{mM}$ ) from ICP-OES assay and the estimated Gd concentration (in $\mathrm{mM}$ ) from MR imaging R1 maps (black markers).

the $\mathrm{T}^{*}$ images, indicating that perivascular hemorrhaging remained below the limit of MR imaging detection. Among the 29 animals conducted histological examinations (with $1720 \mathrm{H} \& \mathrm{E}$ slides), a total of 9.5\% appeared erythrocyte extravasations but still within a controlled and secure scale, ${ }^{3,18}$ and a low incidence $(2.3 \%)$ of unacceptable microhemorrhage level was identified only from group 3 and 4 experiments. All TUNEL staining was negative, suggesting that FUS did not cause apoptosis (On-line Table 2). This implies that it is possible to choose FUS exposure conditions that will securely open the BBB in large animals.

\section{DISCUSSION}

Here we demonstrated the successful use of a neuronavigation system to guide FUS-induced BBB opening. The threshold pressure level required for BBB opening in swine was similar to that reported in mice ${ }^{5,19}$ and primates. ${ }^{11}$ Compared with the MR-guided approach, neuronavigation allows monitoring of BBB opening during the FUS process and significantly reduces costs. A one-time-only MR imaging procedure is sufficient for guidance, which is particularly important for patients undergoing multiple chemotherapeutic treatments, such as brain cancer patients. Neuronavigation has the added advantage of not limiting neurosurgical intervention by MR imaging chamber space or nonmagnetic properties, thus providing high treatment flexibility.

The positional discrepancy of our method $(2.3 \mathrm{~mm})$ was similar to the currently accepted value of neuronavigational surgical-tool guidance (approximately $2 \mathrm{~mm}$ ). ${ }^{14,20}$ Positional errors are known to arise from several sources, ${ }^{14}$ but the most probable source in our study 
is fiducial facial attachment during registration. This could be improved by a skull-implant fiducial, but the process is invasive. Another source of error comes from sonography being diffracted by inhomogeneous bone. The accuracy could likely be increased by the addition of phase correction to a phased array system using CT.

Postoperation MR R1 relaxometry was used to evaluate the success of FUS-induced BBB opening. We found that the GdDTPA concentration was linearly proportional to the R1 relaxation rate. Compared with the qualitative confirmation of $\mathrm{BBB}$ opening obtained from contrast-enhanced T1 images, relaxometry provided a direct and better measurement of Gd-DTPA. Previous small-animal studies have demonstrated that the Gd-DTPA concentration determined from the MR imaging SI change is well correlated with the delivered drug concentration, ${ }^{21,22}$ and our findings revealed the potential of R1 relaxometry to further improve the accuracy and reliability of semiquantitative estimation of local CNS drug delivery.

Tumors are usually bulky (typically $50-90 \mathrm{~mL}$ in volume) and have highly heterogeneous blood-tissue permeability. ${ }^{23,24}$ Large openings in the BBB are therefore desirable for brain tumor treatment in order to affect as many brain tumor cells as possible, including diffusely scattered tumor cells that are often found outside the main contrast enhancement area, adjacent to the FUS BBB-opening site. ${ }^{25} \mathrm{We}$ found that FUS exposure in a $3 \times 3$ pattern successfully created a large BBB-opening region $(>20$ $\mathrm{mm})$ compared with a single FUS exposure $(<5 \mathrm{~mm})$. However, the efficacy of BBB opening is highly dependent on the temporal concentration of microbubbles in the bloodstream. ${ }^{26}$ We therefore controlled the microbubble infusion rate to maintain a constant circulatory bubble concentration during exposure. With a maximum allowable microbubble volume, the overall exposure time has to be limited. A future improvement would be to use a sonography-phased array capable of fast scanning of the focus position via switching the phase difference among elements, thus significantly increasing the total number of FUS exposures.

\section{CONCLUSIONS}

Our successful demonstration of neuronavigation-guided FUS in the swine brain has the potential to become a widely used and flexible medical tool for drug delivery across the BBB into the human brain. The proposed concept could be easily integrated with current neurosurgical interventions. In addition, the design complexity of the FUS system could be reduced by combination with neuronavigation systems already in widespread use, lowering the cost of the overall system. Neuronavigation-guided FUS reduces the requirement of the entire device on MR compatibility and could be used in combination with other anatomic imaging tools. Gd-DTPA relaxometry could be expanded to monitoring or quantifying future in vivo therapeutic agents through imaging, either through direct conjugation to Gd-DTPA or by extrapolation from its relative leakage efficiency compared with that of Gd-DTPA, keeping in mind the strong dependence of BBB permeability on size. The application of a neuronavigation system in guiding FUS for BBB opening will provide a new and more flexible route of accomplishing FUS-enhanced brain drug delivery and is expected to speed up the translation process and widespread use of this technology.

\section{REFERENCES}

1. Brightman MW, Reese TS. Junctions between intimately apposed cell membranes in the vertebrate brain. J Cell Biol 1969;40:648-77

2. Pardridge WM. Drug and gene delivery to the brain: the vascular route. Neuron 2002;36:555-58

3. Hynynen K, McDannold N, Sheikov NA, et al. Local and reversible blood-brain barrier disruption by noninvasive focused ultrasound at frequencies suitable for trans-skull sonications. Neuroimage 2005;24:12-20

4. Hynynen K, McDannold N, Vykhodtseva N, et al. Noninvasive MR imaging-guided focal opening of the blood-brain barrier in rabbits. Radiology 2001;220:640-46

5. Samiotaki G, Vlachos F, Tung YS, et al. A quantitative pressure and microbubble-size dependence study of focused ultrasound-induced blood-brain barrier opening reversibility in vivo using MRI. Magn Reson Med 2012;67:769-77

6. Liu HL, Hsu PH, Chu PC, et al. Magnetic resonance imaging enhanced by superparamagnetic iron oxide particles: usefulness for distinguishing between focused ultrasound-induced blood-brain barrier disruption and brain hemorrhage. J Magn Reson Imaging 2009;29:31-38

7. Cohen ZR, Zaubermann J, Harnof S, et al. Magnetic resonance imaging-guided focused ultrasound for thermal ablation in the brain: a feasibility study in a swine model. Neurosurgery 2007;60:593-600; discussion 600

8. Jagannathan J, Sanghvi NT, Crum LA, et al. High-intensity focused ultrasound surgery of the brain: part 1-A historical perspective with modern applications. Neurosurgery 2009;64:201-10; discussion $210-11$

9. McDannold N, Clement GT, Black P, et al. Transcranial magnetic resonance imaging-guided focused ultrasound surgery of brain tumors: initial findings in 3 patients. Neurosurgery 2010;66:323-32; discussion 332

10. Hynynen K, Clement GT, McDannold N, et al. 500-element ultrasound phased array system for noninvasive focal surgery of the brain: a preliminary rabbit study with ex vivo human skulls. Magn Reson Med 2004;52:100-07

11. Tung YS, Marquet F, Teichert T, et al. Feasibility of noninvasive cavitation-guided blood-brain barrier opening using focused ultrasound and microbubbles in nonhuman primates. Appl Phys Lett 2011;98:163704

12. Xie F, Boska MD, Lof J, et al. Effects of transcranial ultrasound and intravenous microbubbles on blood brain barrier permeability in a large animal model. Ultrasound Med Biol 2008;34:2028-34

13. Liu HL, Chen PY, Yang HW, et al. In vivo MR quantification of superparamagnetic iron oxide nanoparticle leakage during lowfrequency-ultrasound-induced blood-brain barrier opening in swine. J Magn Reson Imaging 2011;34:1313-24

14. Willems PW, van der Sprenkel JW, Tulleken CA, et al. Neuronavigation and surgery of intracerebral tumours. J Neurol 2006;253:1123-36

15. Liu HL, Wai YY, Chen WS, et al. Hemorrhage detection during focused-ultrasound induced blood-brain-barrier opening by using susceptibility-weighted magnetic resonance imaging. Ultrasound Med Biol 2008;34:598-606

16. Li KL, Zhu XP, Waterton J, et al. Improved 3D quantitative mapping of blood volume and endothelial permeability in brain tumors. $J$ Magn Reson Imaging 2000;12:347-57

17. Zhu XP, Li KL, Kamaly-Asl ID, et al. Quantification of endothelial permeability, leakage space, and blood volume in brain tumors using combined $\mathrm{T} 1$ and $\mathrm{T} 2^{\star}$ contrast-enhanced dynamic MR imaging. J Magn Reson Imaging 2000;11:575-85

18. Hynynen K, McDannold N, Martin H, et al. The threshold for brain damage in rabbits induced by bursts of ultrasound in the presence 
of an ultrasound contrast agent (Optison). Ultrasound Med Biol 2003;29:473-81

19. Tung YS, Vlachos F, Choi JJ, et al. In vivo transcranial cavitation threshold detection during ultrasound-induced blood-brain barrier opening in mice. Phys Med Biol 2010;55:6141-55

20. Omay $\mathrm{SB}$, Barnett $\mathrm{GH}$. Surgical navigation for meningioma surgery. J Neurooncol 2010;99:357-64

21. Treat LH, McDannold N, Vykhodtseva N, et al. Targeted delivery of doxorubicin to the rat brain at therapeutic levels using MRI-guided focused ultrasound. Int J Cancer 2007;121:901-07

22. Kinoshita M, McDannold N, Jolesz FA, et al. Noninvasive localized delivery of herceptin to the mouse brain by MRI-guided focused ultrasound-induced blood-brain barrier disruption. Proc Natl Acad Sci U S A 2006;103:11719-23

23. Holodny AI, Nusbaum AO, Festa S, et al. Correlation between the degree of contrast enhancement and the volume of peritumoral edema in meningiomas and malignant gliomas. Neuroradiology $1999 ; 41: 820-25$

24. Tralins KS, Douglas JG, Stelzer KJ, et al. Volumetric analysis of $18 \mathrm{~F}-$ FDG PET in glioblastoma multiforme: prognostic information and possible role in definition of target volumes in radiation dose escalation. J Nucl Med 2002;43:1667-73

25. Kuhn SA, Romeike B, Walter J, et al. Multiplanar MRI-CT fusion neuronavigation-guided serial stereotactic biopsy of human brain tumors: proof of a strong correlation between tumor imaging and histopathology by a new technical approach. J Cancer Res Clin Oncol 2009;135:1293-302

26. Yang FY, Liu SH, Ho FM, et al. Effect of ultrasound contrast agent dose on the duration of focused-ultrasound-induced blood-brain barrier disruption. J Acoust Soc Am 2009;126:3344-49 